\title{
A provisional database for the silicon content of foods in the United Kingdom
}

\author{
J. J. Powell ${ }^{1,2,3 *}$, S. A. McNaughton ${ }^{3}$, R. Jugdaohsingh ${ }^{1}$, S. H. C. Anderson ${ }^{1}$, J. Dear ${ }^{2}$, F. Khot ${ }^{2}$, L. Mowatt ${ }^{1}$, \\ K. L. Gleason ${ }^{2}$, M. Sykes ${ }^{2}$, R. P. H. Thompson ${ }^{1}$, C. Bolton-Smith ${ }^{3}$ and M. J. Hodson ${ }^{4}$ \\ ${ }^{1}$ Gastrointestinal Laboratory, Rayne Institute, St Thomas' Hospital, London SE1 7EH, UK \\ ${ }^{2}$ Department of Nutrition and Dietetics, King's College London, 150 Stamford Street, London SE1 9NN, UK \\ ${ }^{3}$ MRC Human Nutrition Research, Elsie Widdowson Laboratory, Fulbourn Road, Cambridge CB1 9NL, UK \\ ${ }^{4}$ School of Biological and Molecular Sciences, Oxford Brookes University, Oxford OX3 OBP, UK
}

(Received 17 December 2004 - Revised 6 June 2005 - Accepted 7 June 2005)

\begin{abstract}
Si may play an important role in bone formation and connective tissue metabolism. Although biological interest in this element has recently increased, limited literature exists on the $\mathrm{Si}$ content of foods. To further our knowledge and understanding of the relationship between dietary Si and human health, a reliable food composition database, relevant for the UK population, is required. A total of 207 foods and beverages, commonly consumed in the UK, were analysed for Si content. Composite samples were analysed using inductively coupled plasma-optical emission spectrometry following microwave-assisted digestion with nitric acid and $\mathrm{H}_{2} \mathrm{O}_{2}$. The highest concentrations of $\mathrm{Si}$ were found in cereals and cereal products, especially less refined cereals and oat-based products. Fruit and vegetables were highly variable sources of Si with substantial amounts present in Kenyan beans, French beans, runner beans, spinach, dried fruit, bananas and red lentils, but undetectable amounts in tomatoes, oranges and onions. Of the beverages, beer, a macerated whole-grain cereal product, contained the greatest level of $\mathrm{Si}$, whilst drinking water was a variable source with some mineral waters relatively high in $\mathrm{Si}$. The present study provides a provisional database for the Si content of UK foods, which will allow the estimation of dietary intakes of Si in the UK population and investigation into the role of dietary $\mathrm{Si}$ in human health.
\end{abstract}

Dietary silicon: Food composition: Human health

Si is the second most abundant element in the Earth's crust and although there has been interest in the biological role of Si since the beginning of the century, it is only in the last three decades that it has been suggested as an essential trace element (Carlisle, 1972, 1982; Schwarz \& Milne, 1972). Si may have a role in a number of areas of human physiology and metabolism, especially bone and connective tissue formation (Jugdaohsingh et al. 2004), but possibly also gene expression and cardiovascular health (Schwarz, 1977; Carlisle, 1981; Institute of Medicine, 2001).

$\mathrm{Si}$ is rarely found in its elemental form as it readily reacts with atmospheric $\mathrm{O}_{2}$ and water to produce silicates. These vary in composition from simple soluble orthosilicic acid $\left(\mathrm{Si}\left(\mathrm{OH}_{4}\right) \rightleftarrows\right.$ $\left.\mathrm{Si}(\mathrm{OH})_{3} \mathrm{O}^{-}+\mathrm{H}^{+}\right)$and oligomers through to less soluble colloids and gels, and insoluble solid-phase silicates (Iler, 1979). The latter constitute the vast majority of environmental silicates (for example, soil minerals), although natural waters contain soluble silicates (mostly orthosilicic acid) generally at $1-20 \mathrm{mg} /$ 1. Orthosilicic acid is the most readily absorbed form of $\mathrm{Si}$ and silicate in man (Jugdaohsingh et al. 2002). Plants take up orthosilicic acid from the soil, which is transported through the plant in this form, and is then mostly deposited in cell lumina and walls as solid amorphous silica deposits known as phytoliths. Therefore, in plants and plant-based foods, $\mathrm{Si}$ is present as insoluble phytolithic silica, but intestinal uptake proceeds following luminal hydrolysis to orthosilicic acid (Reffitt et al. 1999). Meat and meat products contain very little Si (Pennington, 1991).
Very little data on the Si content of foods has been reported previously, primarily due to difficulties associated with the analytical procedures required for $\mathrm{Si}$ analysis (van Dyck et al. 2000). Existing published literature concentrates mainly on cereals and cereal products and there are limited data available for fruits and vegetables (Pennington, 1991; Bellia et al. 1994; Jugdaohsingh et al. 2002). Data for UK foods are especially limited (Bowen \& Peggs, 1984).

\section{Methods \\ Food sampling}

A preliminary database for the Si content of foods was created by the principal investigator (J. P.) based upon collation of literature values of the Si content of foods and has been used previously to investigate $\mathrm{Si}$ intakes in the Framingham Heart Study cohorts (Jugdaohsingh et al. 2002). This preliminary database was used to identify food items that contributed most to $\mathrm{Si}$ intake and, in the present study, the number of foods analysed from the main Si-containing food groups was in approximate proportion to their contribution to Si intake in the Framingham Study, although a number of additional factors were also considered. First, a number of food items that are more frequently consumed in the UK than in the USA were identified (for example, porridge, oatcakes); second, alcoholic beverages were analysed in detail as 
Table 1. The silicon content of UK foods analysed by inductively coupled plasma-optical emission spectrometry and comparisons with published literature values (Pennington, 1991)

\begin{tabular}{|c|c|c|c|c|c|c|}
\hline \multirow[b]{3}{*}{ Food description } & & \multirow{3}{*}{$\begin{array}{l}\text { No. of food } \\
\text { samples }^{\star}\end{array}$} & \multicolumn{3}{|c|}{ Present study } & \multirow{3}{*}{$\begin{array}{l}\text { Published values } \\
\text { (mg/100 g) }\end{array}$} \\
\hline & & & \multirow[b]{2}{*}{ mg/portion } & \multicolumn{2}{|c|}{$\mathrm{mg} / 100 \mathrm{~g}$} & \\
\hline & & & & Mean & SD & \\
\hline \multicolumn{7}{|l|}{ Cereals and cereal products } \\
\hline $\begin{array}{l}\text { Biscuits, digestives, } \\
\text { chocolate-covered }\end{array}$ & 2 biscuits (36 g) & & 0.88 & $2 \cdot 44$ & & \\
\hline $\begin{array}{l}\text { Biscuits, savoury, cream } \\
\text { crackers }\end{array}$ & 2 biscuits ( $14 \mathrm{~g}$ ) & & 0.19 & $1 \cdot 34$ & & 8 \\
\hline Biscuits, sweet, cream-filled & 2 biscuits $(24 \mathrm{~g})$ & & 0.25 & 1.05 & & \\
\hline $\begin{array}{l}\text { Biscuits, sweet, } \\
\text { shortbread, all-butter }\end{array}$ & 2 biscuits (26g) & & 0.31 & $1 \cdot 19$ & & 3 \\
\hline Bran flakes & 1 medium portion $(30 \mathrm{~g})$ & & 1.45 & $4 \cdot 82$ & & \\
\hline Bread, brown & 1 medium slice, large loaf $(36 \mathrm{~g})$ & & $2 \cdot 22$ & $6 \cdot 17$ & & 6 \\
\hline Bread, brown, rolls & 1 soft roll $(48 \mathrm{~g})$ & & $1 \cdot 80$ & 3.75 & & \\
\hline Bread, naan & 1 plain $(160 \mathrm{~g})$ & & $2 \cdot 82$ & 1.76 & & \\
\hline Bread, white & 1 medium slice, large loaf $(36 \mathrm{~g})$ & & 0.64 & 1.79 & & \\
\hline Bread, white, rolls & 1 soft roll $(45 \mathrm{~g})$ & & $1 \cdot 68$ & 3.73 & & \\
\hline Bread, wholemeal & 1 medium slice, large loaf $(36 \mathrm{~g})$ & & 1.72 & 4.78 & & \\
\hline Bread, wholemeal, rolls & 1 soft roll $(48 \mathrm{~g})$ & & 1.30 & $2 \cdot 71$ & & \\
\hline $\begin{array}{l}\text { Breads, buttery (for example, } \\
\text { croissants or brioche) }\end{array}$ & 1 croissant $(60 \mathrm{~g})$ & & 1.37 & $2 \cdot 29$ & & \\
\hline Cornflakes & 1 medium portion $(30 \mathrm{~g})$ & & 0.56 & $1 \cdot 88$ & & 4 \\
\hline $\begin{array}{l}\text { Cornflakes, honey and } \\
\text { nut-coated }\end{array}$ & 1 medium portion $(30 \mathrm{~g})$ & & 0.93 & $3 \cdot 10$ & & \\
\hline Couscous & 3 tablespoons $(99 \mathrm{~g})$ & & 0.54 & 0.55 & & \\
\hline Crumpets & 1 crumpet $(40 \mathrm{~g})$ & & 0.66 & 1.65 & & \\
\hline Currant buns & 1 individual $(60 \mathrm{~g})$ & & $2 \cdot 36$ & 3.93 & & \\
\hline Flour, wheat, white & 1 tablespoon $(20 \mathrm{~g})$ & & 0.86 & $4 \cdot 29$ & & \\
\hline Flour, wheat, wholemeal & 1 tablespoon $(20 \mathrm{~g})$ & & 0.61 & 3.04 & & \\
\hline Granola cereals & 3 tablespoons $(60 \mathrm{~g})$ & & $7 \cdot 35$ & $12 \cdot 25$ & & \\
\hline High-fibre wheat bran cereal & 4 tablespoons $(28 \mathrm{~g})$ & & $3 \cdot 20$ & 11.42 & & \\
\hline Muesli, Swiss-style & $\frac{1}{2} 4 \mathrm{oz}$ cup $(50 \mathrm{~g})$ & & $2 \cdot 80$ & 5.59 & & \\
\hline $\begin{array}{l}\text { Pasta, fusilli twists, white, } \\
\text { cooked }\end{array}$ & 1 medium portion $(230 \mathrm{~g})$ & & $1 \cdot 86$ & 0.81 & & \\
\hline Pasta, spaghetti, white, boiled & 1 medium portion $(220 \mathrm{~g})$ & & 1.45 & 0.66 & & \\
\hline $\begin{array}{l}\text { Pasta, spaghetti, whole-wheat, } \\
\text { boiled }\end{array}$ & 1 medium portion $(220 \mathrm{~g})$ & & $2 \cdot 84$ & $1 \cdot 29$ & & \\
\hline Pasta, tricolore, cooked & 1 medium portion $(230 \mathrm{~g})$ & & 3.63 & 1.58 & & \\
\hline Pasta, white, egg, cooked & 1 medium portion $(230 \mathrm{~g})$ & & 1.43 & 0.62 & & \\
\hline $\begin{array}{l}\text { Pasta, white, macaroni } \\
\text { or penne, boiled }\end{array}$ & 1 medium portion $(230 \mathrm{~g})$ & & $2 \cdot 28$ & 0.99 & & \\
\hline Pearl barley, boiled & 1 tablespoon $(20 \mathrm{~g})$ & & 0.37 & 1.84 & & $9 \cdot 0$ (raw) \\
\hline Polenta & 1 tablespoon $(33 \mathrm{~g})$ & & 0.11 & 0.34 & & \\
\hline Porridge oats, dry & 2 tablespoons $(30 \mathrm{~g})$ & 3 & 3.42 & 11.39 & 4.25 & 13.0 (rolled oats) \\
\hline Puffed wheat & 2 tablespoons $(6 \mathrm{~g})$ & & 0.13 & $2 \cdot 19$ & & \\
\hline Rice cereal, chocolate-covered & 1 medium portion $(30 \mathrm{~g})$ & & 1.51 & 5.02 & & \\
\hline Rice cereal, toasted and crisped & 1 medium portion $(30 \mathrm{~g})$ & & 0.94 & 3.13 & & \\
\hline Rice, Arborio & 3 heaped tablespoons $(120 \mathrm{~g})$ & & 1.06 & 0.88 & & \\
\hline Rice, basmati, boiled & 3 heaped tablespoons $(120 \mathrm{~g})$ & & $1 \cdot 18$ & 0.98 & & \\
\hline Rice, brown, boiled & 3 heaped tablespoons $(120 \mathrm{~g})$ & & 4.51 & 3.76 & & \\
\hline Rice, jasmine, boiled & 3 heaped tablespoons $(120 \mathrm{~g})$ & & 0.92 & 0.77 & & \\
\hline $\begin{array}{l}\text { Rice, white, boil in the } \\
\text { bag, boiled }\end{array}$ & 3 heaped tablespoons $(120 \mathrm{~g})$ & & $1 \cdot 32$ & $1 \cdot 10$ & & \\
\hline Rice, white, easy-cook, boiled & 3 heaped tablespoons $(120 \mathrm{~g})$ & & $2 \cdot 34$ & 1.95 & & \\
\hline Rice, white, long-grain, boiled & 3 heaped tablespoons $(120 \mathrm{~g})$ & & $2 \cdot 23$ & $1 \cdot 86$ & & \\
\hline Rice, white, short-grain, boiled & 3 heaped tablespoons $(120 \mathrm{~g})$ & & $1 \cdot 18$ & 0.98 & & \\
\hline $\begin{array}{l}\text { Shredded wholegrain wheat } \\
\text { cereal biscuits }\end{array}$ & 2 biscuits ( $45 \mathrm{~g})$ & & 0.60 & $1 \cdot 34$ & & \\
\hline Wheat and rice cereal flakes & 1 medium portion $(30 \mathrm{~g})$ & & $1 \cdot 38$ & $4 \cdot 60$ & & \\
\hline Wheat cereal biscuits & 2 biscuits ( $40 \mathrm{~g})$ & & $2 \cdot 10$ & $5 \cdot 25$ & & \\
\hline Wheat bran & 2 tablespoons (14 g) & 3 & 1.54 & $10 \cdot 98$ & 9.03 & $67 \cdot 0,26 \cdot 0$ \\
\hline \multicolumn{7}{|l|}{ Fruit } \\
\hline $\begin{array}{l}\text { Apples, cooking, fresh, } \\
\text { peeled, cooked }\end{array}$ & 1 average portion $(85 \mathrm{~g})$ & & 1.59 & 1.87 & & \\
\hline
\end{tabular}




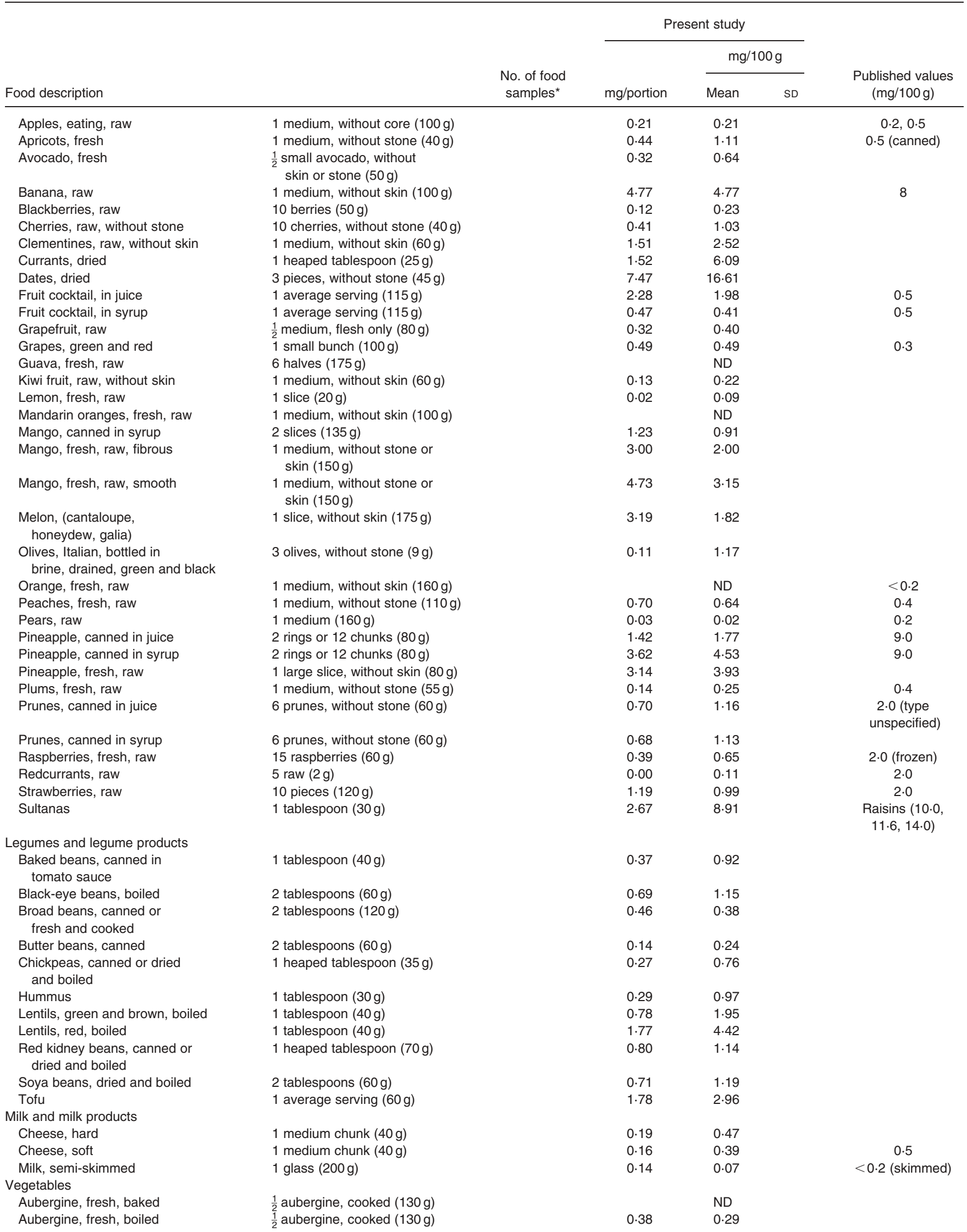




\begin{tabular}{|c|c|c|c|c|c|c|}
\hline \multirow{2}{*}{ Food description } & & \multirow{2}{*}{$\begin{array}{l}\text { No. of food } \\
\text { samples* }\end{array}$} & \multicolumn{3}{|c|}{ Present study } & \multirow{2}{*}{$\begin{array}{l}\text { Published values } \\
\text { (mg/100 g) }\end{array}$} \\
\hline & & & $\mathrm{mg} /$ portion & \multicolumn{2}{|c|}{$\mathrm{mg} / 100 \mathrm{~g}$} & \\
\hline $\begin{array}{l}\text { Beans, green or French, } \\
\text { fresh, boiled }\end{array}$ & 1 medium serving $(90 \mathrm{~g})$ & & $7 \cdot 86$ & $8 \cdot 73$ & & $\begin{array}{c}10 \cdot 0,43.9 \text { (raw); } \\
5.0 \text { (frozen) }\end{array}$ \\
\hline Beans, runner, fresh, boiled & 1 medium serving $(90 \mathrm{~g})$ & & $4 \cdot 73$ & $5 \cdot 25$ & & \\
\hline Broccoli, green, fresh, boiled & 1 medium serving $(85 \mathrm{~g})$ & & 0.66 & 0.78 & & 0.5 (raw) \\
\hline Brussels sprouts, fresh, boiled & 9 sprouts $(90 \mathrm{~g})$ & & & ND & & \\
\hline Cabbage, red, fresh, raw & 1/6th small cabbage $(90 \mathrm{~g})$ & & 0.25 & 0.28 & & $<0.2$ \\
\hline Cabbage, regular, fresh, raw & 1/6th small cabbage $(90 \mathrm{~g})$ & & & ND & & $\begin{array}{c}0.5,0 \cdot 7,0 \cdot 8 \\
4 \cdot 0,17 \cdot 1\end{array}$ \\
\hline Cabbage, savoy, fresh, raw & 1/6th small cabbage $(90 \mathrm{~g})$ & & 0.64 & 0.71 & & \\
\hline Cabbage, white, fresh, raw & 1/6th small cabbage $(90 \mathrm{~g})$ & & 0.48 & 0.53 & & \\
\hline $\begin{array}{l}\text { Carrots, new and old, } \\
\text { fresh, raw }\end{array}$ & 1 tablespoon $(40 \mathrm{~g})$ & & 0.04 & $0 \cdot 10$ & & \\
\hline Cauliflower, fresh, boiled & 6 florets $(60 \mathrm{~g})$ & & 0.13 & 0.22 & & 0.5 \\
\hline Celery, fresh, raw & 1 stick $(30 \mathrm{~g})$ & & 0.09 & 0.29 & & 5.5 \\
\hline Coriander, fresh, raw & $\frac{1}{2}$ tablespoon $(2 \mathrm{~g})$ & & 0.17 & 8.42 & & \\
\hline Courgette, fresh, boiled & 1 medium courgette $(100 \mathrm{~g})$ & & 0.95 & 0.95 & & \\
\hline Cucumber, fresh, raw & 1 inch piece $(60 \mathrm{~g})$ & & 1.52 & 2.53 & & $3 \cdot 0,4 \cdot 6$ \\
\hline $\begin{array}{l}\text { Kenyan or fine beans, } \\
\text { fresh, boiled }\end{array}$ & 1 medium serving $(90 \mathrm{~g})$ & & $9 \cdot 00$ & $10 \cdot 00$ & & \\
\hline Leeks, fresh, boiled & $\frac{1}{2}$ medium leek $(80 \mathrm{~g})$ & & 0.28 & 0.35 & & 6.0 (raw) \\
\hline Lettuce, iceberg, fresh, raw & $\begin{array}{l}1 \text { average serving in } \\
\text { salad }(80 \mathrm{~g})\end{array}$ & & 0.92 & $1 \cdot 15$ & & 2 \\
\hline Lettuce, lamb's, fresh, raw & $\begin{array}{l}1 \text { average serving in } \\
\text { salad }(30 \mathrm{~g})\end{array}$ & & 0.54 & $1 \cdot 81$ & & 2 \\
\hline Lettuce, little gem, fresh, raw & $\begin{array}{l}1 \text { average serving in } \\
\text { salad }(30 \mathrm{~g})\end{array}$ & & 0.33 & $1 \cdot 11$ & & 2 \\
\hline Lettuce, rocket, fresh, raw & $\begin{array}{l}1 \text { average serving in } \\
\text { salad }(30 \mathrm{~g})\end{array}$ & & 0.83 & $2 \cdot 78$ & & \\
\hline Mange-tout, fresh, boiled & 1 medium serving $(90 \mathrm{~g})$ & & 0.69 & 0.77 & & \\
\hline Marrow, fresh, raw & 1 average serving $(65 \mathrm{~g})$ & 3 & 3.04 & 4.68 & 0.01 & \\
\hline Onion, normal, fresh, raw & $\frac{1}{2}$ small onion $(30 \mathrm{~g})$ & & & ND & & 0.5 \\
\hline Onions, white, fresh, raw & $\frac{1}{2}$ small onion $(30 \mathrm{~g})$ & & & ND & & 0.5 \\
\hline Parsnips, fresh, peeled, boiled & 1 tablespoon $(50 \mathrm{~g})$ & & $1 \cdot 13$ & $2 \cdot 26$ & & 0.5 (raw) \\
\hline Peas, fresh, boiled & 2 tablespoon $(60 \mathrm{~g})$ & & $0 \cdot 11$ & 0.19 & & 0.4 (raw) \\
\hline Peas, frozen, boiled & 2 tablespoon $(60 \mathrm{~g})$ & & 1.05 & 1.75 & & 0.3 (frozen) \\
\hline Peppers, green, fresh, raw & $\frac{1}{4}$ whole pepper $(40 \mathrm{~g})$ & & $0 \cdot 12$ & 0.29 & & \\
\hline Peppers, red, fresh, raw & $\frac{1}{4}$ whole pepper $(40 \mathrm{~g})$ & & 0.09 & 0.22 & & 0.2 \\
\hline Petit pois, frozen, boiled & 2 tablespoon $(60 \mathrm{~g})$ & & 0.32 & 0.53 & & \\
\hline Potato, fresh, peeled, boiled & 1 medium potato $(50 \mathrm{~g})$ & & 0.17 & 0.34 & & $<0.5$ \\
\hline Potato, oven chips, cooked & 1 medium serving $(165 \mathrm{~g})$ & & 0.68 & 0.41 & & \\
\hline Potatoes, new, peeled, boiled & 1 medium potato $(40 \mathrm{~g})$ & & 0.22 & 0.56 & & $0.2($ raw $)$ \\
\hline Radish, fresh & 5 medium radishes $(40 \mathrm{~g})$ & & $0 \cdot 16$ & 0.39 & & $0 \cdot 1,2 \cdot 0,5 \cdot 1$ \\
\hline Salad, mixed leaves, fresh, raw & $\begin{array}{l}1 \text { average serving } \\
\text { in salad }(80 \mathrm{~g})\end{array}$ & & 1.41 & $1 \cdot 76$ & & \\
\hline Spinach, fresh, boiled & 2 tablespoons $(80 \mathrm{~g})$ & & $4 \cdot 10$ & $5 \cdot 12$ & & $3 \cdot 0,5 \cdot 0$ (raw) \\
\hline Spring greens, fresh, boiled & 1 average serving $(75 \mathrm{~g})$ & & 1.36 & 1.81 & & \\
\hline Spring onions, fresh, raw & 1 spring onion $(10 \mathrm{~g})$ & & 0.24 & 2.43 & & \\
\hline Swede, fresh, boiled & 1 medium portion $(60 \mathrm{~g})$ & & 1.30 & $2 \cdot 17$ & & \\
\hline $\begin{array}{l}\text { Sweet potato, fresh, peeled, } \\
\text { boiled }\end{array}$ & 2 medium potatoes $(130 \mathrm{~g})$ & & 0.35 & 0.27 & & \\
\hline Sweetcorn, canned, drained & 2 tablespoons $(60 \mathrm{~g})$ & & 0.28 & 0.46 & & \\
\hline $\begin{array}{l}\text { Sweetcorn, on the cob, } \\
\text { fresh, boiled }\end{array}$ & $\begin{array}{l}1 \text { corn on cob, kernels } \\
\text { only }(125 \mathrm{~g})\end{array}$ & & 0.48 & 0.38 & & \\
\hline Tomatoes, fresh, raw & 1 medium tomato $(85 \mathrm{~g})$ & & & ND & & $<0.2,0.13,6.1$ \\
\hline Turnips, fresh, peeled, boiled & 1 whole $(110 \mathrm{~g})$ & & 1.00 & 0.91 & & $12 \cdot 0$ (raw) \\
\hline Yam, fresh, peeled, boiled & $\begin{array}{l}\text { Equivalent to } 1 \text { medium } \\
\text { potato }(130 \mathrm{~g})\end{array}$ & & 0.48 & 0.37 & & \\
\hline \multicolumn{7}{|l|}{ Snack foods } \\
\hline Chocolate, milk & 1 average bar $(50 \mathrm{~g})$ & & 0.51 & 1.01 & & 1 \\
\hline Corn chips† & 1 packet $(50 \mathrm{~g})$ & & $2 \cdot 22$ & 4.44 & & \\
\hline Crisps & 1 small packet $(30 \mathrm{~g})$ & & 0.14 & 0.47 & & \\
\hline \multicolumn{7}{|l|}{ Nuts and seeds } \\
\hline Brazil nuts & 6 whole $(20 \mathrm{~g})$ & & 0.06 & 0.28 & & \\
\hline
\end{tabular}




\begin{tabular}{|c|c|c|c|c|c|c|}
\hline \multirow[b]{3}{*}{ Food description } & & \multirow{3}{*}{$\begin{array}{l}\text { No. of food } \\
\text { samples* }\end{array}$} & \multicolumn{3}{|c|}{ Present study } & \multirow{3}{*}{$\begin{array}{l}\text { Published values } \\
\text { (mg/100 g) }\end{array}$} \\
\hline & & & \multirow[b]{2}{*}{ mg/portion } & \multicolumn{2}{|c|}{$\mathrm{mg} / 100 \mathrm{~g}$} & \\
\hline & & & & Mean & SD & \\
\hline Cashew nuts, roasted & 1 small bag $(25 \mathrm{~g})$ & & 0.15 & 0.60 & & \\
\hline $\begin{array}{l}\text { Groundnuts, roasted } \\
\text { and salted }\end{array}$ & 1 small bag $(25 \mathrm{~g})$ & & 0.06 & $0 \cdot 24$ & & 4.7 \\
\hline \multicolumn{7}{|l|}{ Beverages†‡ } \\
\hline Bitter lemon & 1 can $(337 \mathrm{~g})$ & & 0.47 & $0 \cdot 14$ & & \\
\hline $\begin{array}{l}\text { Carbonated beverages, other, } \\
\text { canned }\end{array}$ & 1 can $(343 \mathrm{~g})$ & & 0.41 & $0 \cdot 12$ & & $0.3,0.6,0.3$ \\
\hline $\begin{array}{l}\text { Cocoa, powdered, made up } \\
\text { with water }\end{array}$ & 1 mug $(260 \mathrm{~g})$ & & 1.04 & 0.40 & & \\
\hline $\begin{array}{l}\text { Coffee, instant, decaffeinated, } \\
\text { made up with water }\end{array}$ & 1 mug $(260 \mathrm{~g})$ & & 0.62 & $0 \cdot 24$ & & \\
\hline Cola, canned & 1 can $(343 \mathrm{~g})$ & & 0.69 & 0.20 & & 0.2 \\
\hline Cola, low-energy, canned & 1 can $(343 \mathrm{~g})$ & & 0.38 & $0 \cdot 11$ & & \\
\hline $\begin{array}{l}\text { Drinking chocolate, powdered, } \\
\text { low-energy, made up with water }\end{array}$ & $1 \mathrm{mug}(260 \mathrm{~g})$ & & $10 \cdot 3$ & 3.96 & & \\
\hline $\begin{array}{l}\text { Drinking chocolate, powdered, } \\
\text { made up with water }\end{array}$ & 1 mug $(260 \mathrm{~g})$ & & $1 \cdot 27$ & 0.49 & & \\
\hline Energy drinks, canned & 1 slim can (260 g) & & 0.21 & 0.08 & & \\
\hline Fruit drink, carbonated, canned & 1 can $(343 \mathrm{~g})$ & & 0.38 & 0.11 & & \\
\hline $\begin{array}{l}\text { Fruit drink, carbonated, } \\
\text { low-energy, canned }\end{array}$ & 1 can $(343 \mathrm{~g})$ & & 1.03 & 0.30 & & \\
\hline Fruit juice drink, carton & 1 carton $(260 \mathrm{~g})$ & & 0.39 & $0 \cdot 15$ & & \\
\hline $\begin{array}{l}\text { Fruit juice drink, } \\
\text { low-energy, carton }\end{array}$ & 1 carton $(260 \mathrm{~g})$ & & 0.26 & $0 \cdot 10$ & & \\
\hline Juice, apple, fresh & 1 glass $(160 \mathrm{~g})$ & & 0.08 & 0.05 & & \\
\hline Juice, apple, long-life & 1 glass $(160 \mathrm{~g})$ & & 0.42 & 0.26 & & \\
\hline Juice, orange, fresh & 1 glass $(160 \mathrm{~g})$ & & $0 \cdot 21$ & 0.13 & & \\
\hline Juice, orange, long-life & 1 glass $(160 \mathrm{~g})$ & & 0.19 & $0 \cdot 12$ & & \\
\hline Lemonade, canned & 1 can $(337 \mathrm{~g})$ & & 0.64 & $0 \cdot 19$ & & \\
\hline Lemonade, low-energy, canned & 1 can $(337 \mathrm{~g})$ & & 0.40 & 0.12 & & \\
\hline $\begin{array}{l}\text { Malt-based drink, low-fat, } \\
\text { powdered, made up with water }\end{array}$ & 1 mug $(260 \mathrm{~g})$ & & $3 \cdot 25$ & $1 \cdot 25$ & & \\
\hline $\begin{array}{l}\text { Malt-based drink, powdered, } \\
\text { made up with water }\end{array}$ & 1 mug $(260 \mathrm{~g})$ & & 3.93 & 1.51 & & \\
\hline $\begin{array}{l}\text { Milk shake, powdered, } \\
\text { made up with water }\end{array}$ & 1 mug $(260 \mathrm{~g})$ & & 0.52 & $0 \cdot 20$ & & \\
\hline $\begin{array}{l}\text { Tea, black, decaffeinated, } \\
\text { infusion }\end{array}$ & 1 mug $(260 \mathrm{~g})$ & & 0.78 & 0.30 & & \\
\hline Tea, camomile, infusion & 1 mug $(260 \mathrm{~g})$ & & 0.68 & 0.26 & & \\
\hline $\begin{array}{l}\text { Tea, black, ordinary, } \\
\text { infusion, leaves }\end{array}$ & 1 mug (260 g) & & 0.33 & 0.81 & & 0.2 \\
\hline $\begin{array}{l}\text { Tea, black, ordinary, } \\
\text { infusion, tea bag }\end{array}$ & 1 mug (260 g) & & 0.31 & $0 \cdot 86$ & & 0.2 \\
\hline $\begin{array}{l}\text { Water, mineral, non-English, } \\
\text { sparkling }\end{array}$ & 1 bottle $(330 \mathrm{~g})$ & 3 & $2 \cdot 31$ & $0 \cdot 70$ & 0.51 & \\
\hline $\begin{array}{l}\text { Water, mineral or } \\
\text { spring water, sparkling }\end{array}$ & 1 bottle $(330 \mathrm{~g})$ & 3 & 1.29 & 0.39 & $0 \cdot 14$ & \\
\hline $\begin{array}{l}\text { Water, mineral or } \\
\text { spring, still }\end{array}$ & 1 bottle $(330 \mathrm{~g})$ & 3 & $1 \cdot 78$ & 0.54 & 0.49 & \\
\hline Water, plain, bottled & 1 bottle $(330 \mathrm{~g})$ & & 1.65 & 0.50 & & \\
\hline Water, soda & 1 can $(170 \mathrm{~g})$ & & 0.15 & 0.09 & & \\
\hline Water, tap & 1 glass $(200 \mathrm{~g})$ & 4 & 0.50 & 0.25 & $0 \cdot 11$ & $\begin{array}{c}0.34 \\
\text { (Denver, USA); } 0.02 \\
\text { (Wales, UK) }\end{array}$ \\
\hline Water, tonic & 1 can $(170 \mathrm{~g})$ & & 0.37 & 0.22 & & \\
\hline \multicolumn{7}{|l|}{ Alcoholic beverages§ } \\
\hline Bitter or ale, bottled & 1 bottle $(300 \mathrm{~g})$ & 8 & $5 \cdot 49$ & $1 \cdot 83$ & 0.45 & $\begin{array}{c}3.0(<2.25 \% \\
\text { alcohol })\end{array}$ \\
\hline Bitter or ale, canned & 1 small can $(333 \mathrm{~g})$ & 3 & $3 \cdot 80$ & $1 \cdot 14$ & 0.38 & $\begin{array}{l}3.0(3-3.7 \% \\
\text { alcohol })\end{array}$ \\
\hline
\end{tabular}




\begin{tabular}{|c|c|c|c|c|c|c|}
\hline \multirow[b]{3}{*}{ Food description } & & \multirow{3}{*}{$\begin{array}{l}\text { No. of food } \\
\text { samples* }\end{array}$} & \multicolumn{3}{|c|}{ Present study } & \multirow{3}{*}{$\begin{array}{l}\text { Published values } \\
\text { (mg/100 g) }\end{array}$} \\
\hline & & & \multirow[b]{2}{*}{ mg/portion } & \multicolumn{2}{|c|}{$\mathrm{mg} / 100 \mathrm{~g}$} & \\
\hline & & & & Mean & SD & \\
\hline Bitter or ale, draft & 1 pint $(574 \mathrm{~g})$ & 5 & $12 \cdot 60$ & $2 \cdot 19$ & 0.60 & $\begin{array}{c}6.0(3.7-4.5 \% \\
\text { alcohol) }\end{array}$ \\
\hline Cider & 1 pint $(574 \mathrm{~g})$ & 3 & $2 \cdot 30$ & 0.40 & 0.07 & \\
\hline Lager, canned & 1 small can $(333 \mathrm{~g})$ & 4 & 5.46 & 1.64 & 0.51 & \\
\hline Lager, draft & 1 pint $(574 \mathrm{~g})$ & 3 & $16 \cdot 30$ & $2 \cdot 84$ & 1.05 & \\
\hline Stout, bottled & 1 bottle $(300 \mathrm{~g})$ & 2 & $5 \cdot 28$ & $1 \cdot 76$ & 0.12 & \\
\hline Stout, canned & 1 small can $(333 \mathrm{~g})$ & 4 & 6.06 & $1 \cdot 82$ & 0.59 & \\
\hline Wine, fortified, port & 1 small glass $(50 \mathrm{~g})$ & 2 & 0.62 & $1 \cdot 24$ & 0.15 & \\
\hline Wine, fortified, sherry & 1 small glass $(50 \mathrm{~g})$ & 3 & 0.63 & 1.26 & 0.06 & \\
\hline Wine, red & 1 glass $(125 \mathrm{~g})$ & 7 & 0.85 & 0.68 & 0.59 & $0.20-2 \cdot 35$ \\
\hline Wine, rosé & 1 glass $(125 \mathrm{~g})$ & 3 & 2.89 & $2 \cdot 31$ & 0.84 & 1.99 \\
\hline
\end{tabular}

ND, non-detectable level of Si. For details, see p.810.

${ }^{*}$ For most foods, three samples of the food were used to provide a composite sample. However, for some foods a composite sample was not used and a number of individual foods were analysed; for these the mean and standard deviation are presented together with the number of food samples used.

†These products (particularly low-energy drinking chocolate) may contain non-bioavailable silicate additives leading to a high Si content.

$\ddagger$ Mean Si content of water used to dilute was $0.196 \mathrm{mg} / 100 \mathrm{~g}$

$\S$ Si levels were not related alcohol content in the present study or a previous study (Sripanyakorn et al. 2004).

recent data showed that some of these, such as beer, may be especially high in Si (Sripanyakorn et al. 2004). Third, a few plant-based foods that had not been adequately investigated in the literature, but could contain substantial amounts of $\mathrm{Si}$, were also included for analysis (for example, nuts). Finally, drinking water, which may be a significant source of dietary $\mathrm{Si}$ and is used in beverages (for example, tea, coffee and cold drinks) and in homemade soups and sauces, was analysed for use with water intake and recipe calculations. Processed foods were not included in the analysis as they may have Si added in a relatively non-bioavailable form, typically as particulate silicates and aluminosilicates (Dobbie \& Smith, 1982; Institute of Medicine, 2001), and their natural Si content can be calculated from knowledge of the ingredients. Meat, poultry, fish and seafood contain very little $\mathrm{Si}$ and therefore were not investigated here (Pennington, 1991).

Selected foods (Table 1) were bought from supermarkets and greengrocers at three different locations in Southeast England (i.e. each food was purchased in triplicate), with selection based upon supermarket availability (i.e. popularity of product) and the frequency of ingestion of the food item. Equal amounts of each food from the three sources were combined, taking care to form a representative composite (for example, outer and inner leaves of green vegetables at an appropriate ratio) and then homogenised in a food blender. Some foods were analysed without compositing, in order to observe within-food variations of Si content, but at least three different samples of the same food type were then analysed. Foods were generally prepared in their 'as eaten' form and a single large batch of tap water of known Si content was used to boil foods where appropriate.

\section{Chemical analysis}

Throughout the study no glassware was used, and to avoid excessive skin contact, powder-free, polythene gloves were used on all occasions. Samples were digested and analysed in the Gastrointestinal Laboratory (St Thomas' Hospital, London, UK). Sample preparation and analysis was performed in a class $\mathrm{J}$ clean air room. Homogenised samples were placed into $\mathrm{Si}$-free $10 \mathrm{ml}$ polypropylene transport tubes (Medfor Products, Farnborough, UK). Acid-assisted microwave digestion was conducted using a Milestone Ethos Plus Microwave Digestion System (Milestone Analytical UK Ltd, Northwich, Cheshire, UK) using nitric acid (65\%, w/v, ANALAR grade; Merck, West Drayton, UK) and $\mathrm{H}_{2} \mathrm{O}_{2}$ (30\%, w/v, ARISTAR grade; Merck, West Drayton, UK). Approximately $0.25 \mathrm{~g}$ homogenised sample was added to a digestion vessel with $10 \mathrm{ml}$ nitric acid and $1 \mathrm{ml} \mathrm{H}_{2} \mathrm{O}_{2}$ and the vessel sealed, heated (ramped) to $200^{\circ} \mathrm{C}$ over $10 \mathrm{~min}$ and maintained at $200^{\circ} \mathrm{C}$ for $15 \mathrm{~min}$. Sample blanks were similarly prepared using $10 \mathrm{ml}$ nitric acid (69\%, w/v) and $1 \mathrm{ml} \mathrm{H}_{2} \mathrm{O}_{2}(30 \%$, w/v). Digested samples and blanks were diluted with approximately $33 \mathrm{ml}$ ultrahigh purity water $(18 \mathrm{M} \Omega / \mathrm{cm}$; Elga Ltd, High Wycombe, UK) and total final volumes, assessed accurately by weight, were recorded before analysis. Acid digestion was not needed for most fluids, apart from viscous samples (for example, port and sherry), as the majority could be analysed directly or with simple dilution with ultra-high purity water. $\mathrm{Si}$ analysis was performed using an inductively coupled plasma-optical emission spectrometer (Jobin-Yvon JY24; Instruments SA, Longjumeau, France) with a V-groove nebuliser and Scott-type double-pass spray chamber at $251.611 \mathrm{~nm}$ as previously described (Jugdaohsingh et al. 2002). Analysis was by peak profile with a window size of $0.1 \mathrm{~nm}$, fifty-four increments per profile, an integration time of $0.5 \mathrm{~s}$ and sample flow rate was $1 \mathrm{ml} / \mathrm{min}$. Si standards were prepared from a stock ICP-DCP standard solution (1000 mg Si/l; Aldrich Chemical Company, Gillingham, UK) in matrix-matched solutions (i.e. sample-based standards). Standard reference materials do not exist for $\mathrm{Si}$ in foods or clinical samples, so spiking and recovery experiments were used as before for quality assurance (Sripanyakorn et al. 2004). Under these conditions of microwave digestion, certain solid-phase silicates, especially crystalline silica and silicates, may undergo only partial digestion and require hydrofluoric acid for full dissolution. However, dietary $\mathrm{Si}$ is not expected to occur in the form of crystalline silicates, while the conditions of microwave digestion far exceed 
gastrointestinal digestion. Therefore, any small amounts of undissolved silicates are unlikely to be absorbed in the gastrointestinal tract.

To ensure that Si detected in the sample was from the digested food, and not from 'noise' or background, a sample detection limit was established. The mean $\pm 2 \mathrm{SD}$ of the $\mathrm{Si}$ content of the sample blanks was determined on each day of analysis. These values were then used to calculate the minimum observable value for a 'positive result' in the digested food samples. If the food sample had a reading of less than the mean $+2 \mathrm{SD}$ of the blanks, then the Si content of the food was considered undetectable. Mean Si in the sample blanks was 47.8 (SD 16.4) parts per billion translating to a mean sample detection limit (wet weight), for the digested and diluted samples, of $14.3 \mathrm{~g} \mathrm{Si}$ per $\mathrm{g}$ sample. Further analytical details are available in the Food Standards Agency Report (Powell \& Bolton-Smith, 2003).

Precision of analysis depends on sample type (i.e. matrix). Precision was less than $3 \%$ for most cereal foods, 4.66 (range 0-22.6) \% for fruit and vegetables and 3.34 (range 0$16 \cdot 2) \%$ for beverages. Overall, as expected, precision was greatest in samples with high Si content and poorest in those with low content. This is reported in detail in the Food Standards Agency Report (Powell \& Bolton-Smith, 2003).

The Si content of each food is expressed per $100 \mathrm{~g}$ edible portion and by typical portion size in the UK (Davies \& Dickerson, 1991; Ministry of Agriculture Fisheries and Foods, 1993). For foods where multiple samples were analysed rather than a composite sample, the mean and standard deviation of Si content of the food samples is provided.

\section{Results}

Table 1 presents the Si content of the 207 foods and beverages analysed. Comparisons with the published literature are also presented in Table 1 where relevant or appropriate values exist.

Almost half of the foods analysed (ninety-five items) had low levels of $\mathrm{Si}(<1.0 \mathrm{mg} / 100 \mathrm{~g})$ and there were ten digested foods that had levels of $\mathrm{Si}$ that were indistinguishable from background levels (i.e. sample blanks nitric acid and $\mathrm{H}_{2} \mathrm{O}_{2}$ alone; see p. 809): A total of eighteen foods contained high levels of $\mathrm{Si}(>5.0 \mathrm{mg} /$ $100 \mathrm{~g}$ ). The majority of these foods (eleven items) were from the breads and cereals group and related cereal products, with oat-containing products having the highest levels of all cereals and oat bran having the highest Si content of all items analysed. The remaining items were from the vegetables group (namely, Kenyan beans, green beans, runner beans, spinach and coriander) or were dried fruits. When results were expressed in terms of $\mathrm{Si}$ content per portion, those foods with a high Si content were predominantly beers (seven out of twelve items).

There was considerable variation in the Si content of vegetables, legumes and fruits. With respect to vegetables and legumes, as noted earlier, the richest sources of Si were Kenyan beans, French beans and runner beans, spinach and red lentils. The majority of other vegetables contained low or undetectable amounts of $\mathrm{Si}(<1.0 \mathrm{mg}$ per $100 \mathrm{~g}$ ). With respect to fresh fruits, the highest levels of Si were found in the banana, mango and pineapple.

Of the non-alcoholic beverages, drinking chocolate provided the highest mean $\mathrm{Si}$ content, although this is probably due to the addition of particulate silicate additives rather than a high natural Si content (Lomer et al. 2004). Tap water contained more Si per $100 \mathrm{~g}$ than the carbonated soft drinks while sparkling mineral waters contained more $\mathrm{Si}$ than the still water samples. The Si content of juices (for example, orange, apple and pineapple juice) corresponded to the content of the fresh form of the fruit with apple and orange juices having low levels and pineapple juice showing higher levels. With respect to the alcoholic beverages, beer had higher mean Si content than wine, and cider had the lowest Si content, again corresponding to the $\mathrm{Si}$ contents of the major raw materials, namely barley, grapes and apples respectively.

The remaining food groups, including the milk and milk products and the nuts and seeds, all contained low levels of Si.

\section{Discussion}

Data presented in the present paper represent the first available food composition database for the Si content of UK foods. A total of 207 different foods were analysed covering all the major potential Si-containing foods and food groups for the UK population. Almost half of all the foods analysed had a low Si content and a small number had levels that were undetectable. The highest levels of $\mathrm{Si}$ were found in the cereals, especially less refined cereals and oat-based products. Fruit and vegetables were highly variable sources of $\mathrm{Si}$, with substantial amounts present in green beans, dried dates, bananas and red lentils but undetectable amounts in tomatoes, oranges and onions.

For many plant foods, consideration of the botanical characteristics of the plant may explain their observed Si content. Overall, fruit and vegetables with trichomes or ultrafine hairs on their surface, which are potential locations for Si depositions, tend to have higher Si content. Further evidence supporting the contribution of these fine hairs to the Si content is available from the observed content of the mature seeds. For example, the French bean is consumed either as an immature fruit or pod, which has trichomes present on its surface and a high Si level, or is matured and eaten as the kidney bean (Southgate, 2000) where the pod has been removed resulting in a substantial decrease in the level of Si. Similarly, spinach contains a moderate level of $\mathrm{Si}$, which may also be due to the numerous silicified trichomes on the leaf epidermis. In cereals, it is the silicification of inflorescence bracts, and the siliceous epicarp hairs at the brush end of the mature caryopsis that are responsible for the high $\mathrm{Si}$ levels observed (Hayward \& Parry, 1980; Hodson \& Sangster, 1988). Si has been found along the epicarp hairs of the mature caryopses of wheat, barley and oats, but was most concentrated on the surface and at the tips of the hairs (Bennet \& Parry, 1981; Parry et al. 1984).

Alcoholic beverages, especially the beers and wine, were shown to have a moderate to high Si content. Beer provides a significant amount of absorbable Si obtained from the hot mashing technique that extracts soluble orthosilicic acid from phytolithic silica in the barley husks which adhere to the grain (Sripanyakorn et al. 2004). Due to the relatively high consumption of beer among the UK population (Bellia et al. 1994), beer could contribute substantially to the mean daily $\mathrm{Si}$ intakes. For example, in 1990, consumption of beer in the UK was 194 pints per individual per annum, which would provide a daily Si intake of $6 \mathrm{mg} / \mathrm{indi-}$ vidual (Bellia et al. 1994).

With respect to wine, it is known that $\mathrm{Si}$ is taken up by the grape vines, and some is transferred to the grapes, but little is known about the balance between endogenous $\mathrm{Si}$ and $\mathrm{Si}$ from other sources in determining the final $\mathrm{Si}$ concentration of wine (Blaich \& Grundhofer, 1997). For example, the presence of Si in wine may also be due to the spraying of the grapes with potassium 
silicate to control powdery mildew before harvest or due to the use of clarifying agents, such as bentonite and colloidal silica gel (EC Council, 1999; Cabras \& Angioni, 2000).

Although tap water had a relatively low Si content, it also has the potential to contribute a substantial amount to overall Si intake due to the amount normally consumed directly, as a diluent in other beverages and as an ingredient in many recipes. The Si content of tap water can vary according to the geographical region, with typically high Si levels in hard water areas and low levels in soft water areas (Seaborn \& Nielsen, 1993). In a study carried out in Egypt, bottled water of all brands contained higher levels of Si than tap water (Saleh et al. 2001), which also was reflected in the findings here with higher Si levels in spring and mineral waters. This may well be because tap water treatment (i.e. by Al flocculation) decreases the Si content.

Manufacturing processes may also affect the Si content of the final food item. First, although cereals and related products were shown to have high levels of $\mathrm{Si}$ compared with other food items, the level of Si varied according to the level of refining, which determines how much of the original silicified plant material is preserved in the final food item. Second, the Si content of some fruits and legumes may have also been affected by processing. For example, dates accumulate $\mathrm{Si}$ in substantial quantities but the drying process further increases their Si content due to the loss of water (Holland et al. 1991; Cummings, 1992). Hence, dried fruits in the present study (for example, sultanas), as reported elsewhere in the literature (Pennington, 1991), have relatively high Si levels. In addition, $\mathrm{Si}$ may be added to some dried foods in the form of silica gel $\left(\mathrm{SiO}_{2}\right)$ (Hughes, 1987). The high Si content of red lentils was surprising when compared with related foods such as other legumes consumed as mature seeds or in the fresh form (peas, chick peas, etc.) which all had Si levels below $1 \mathrm{mg} / 100 \mathrm{~g}$, suggesting that drying processes or storage may be responsible for the higher Si content of red lentils. Third, high levels of $\mathrm{Si}$ found in drinking chocolate and corn chips may relate to $\mathrm{Si}$ added as particulate and non-bioavailable silicates during processing, chiefly as anti-caking agents (Expert Group on Vitamins and Minerals, 2003; Lomer et al. 2004).

The Si content of foods analysed in the present study was compared with the published literature where appropriate or relevant data were available. The Si contents of the majority of beverages and cereals were comparable with values available in the published literature, except for three products (white flour, savoury biscuits and Weetabix), with the Si content of plain white flour reported in the literature being nearly double that found in the present study (Table 1). Comparisons between the literature and the present study were less consistent for fruit and vegetables. This may be due to variations in the plant cultivars grown in different countries, geographical variations in soil and water $\mathrm{Si}$ contents used in agriculture, and in variations in $\mathrm{Si}$ content of water used in cooking for cooked items. Finally, banana was shown here and in previous work to have a high $\mathrm{Si}$ content, in contrast to the majority of other fruits, although the reasons for its high Si content are unknown (Pennington, 1991). However, the importance of bananas in contributing to an individual's $\mathrm{Si}$ exposure is questionable, as little is thought to be bioavailable following consumption of the fruit (Jugdaohsingh et al. 2002).

\section{Conclusions}

The present study provides a provisional database for the $\mathrm{Si}$ content of foods based on laboratory analysis of carefully selected
UK foods. This database will allow the estimation of dietary intakes in the UK population and investigation into the role of dietary Si in human health.

\section{Acknowledgements}

The present study was mainly supported by the UK Food Standards Agency (FSA) (project NO5034) and we are especially grateful to Ms Lynn Burns (FSA) for helpful suggestions and guidance throughout. We gratefully acknowledge the support of Dr Alison Tedstone (FSA) and Ms Victoria Targett (FSA). We acknowledge The Wellcome Trust for support of the ICPOES. S. A. was a Wellcome Trust Training Fellow and R. J. is supported by a fellowship from The Frances and Augustus Newman Foundation.

\section{References}

Bellia JP, Birchall JD \& Roberts NB (1994) Beer: a dietary source of silicon. Lancet 343, 235.

Bennet DM \& Parry DW (1981) Electron-probe microanalysis studies of silicon in the epicarp hairs of the caryopses of Hordeum sativum Jess, Avena sativa L, Secale cereale L, and Triticum aestivum L. Ann Bot 48, $645-654$.

Blaich R \& Grundhofer H (1997) Uptake of silica by grapevines from soil and recirculating nutrient solutions. Vitis 36, 161-166.

Bowen HJM \& Peggs A (1984) Determination of the silicon content of food. J Sci Food Agric 35, 1225-1229.

Cabras P \& Angioni A (2000) Pesticide residues in grapes, wine, and their processing products. J Agric Food Chem 48, 967-973.

Carlisle EM (1972) Silicon: an essential element for the chick. Science 178, 619-662.

Carlisle EM (1981) Silicon: a requirement in bone formation independent of vitamin D. Calcif Tissue Int 33, 27-34.

Carlisle EM (1982) The nutritional essentiality of silicon. Nutr Rev 40, $193-198$.

Cummings LS (1992) Illustrated phytoliths from assorted food plants. In Phytolith Systematics - Emerging Issues, pp. 175-192 [G Rapp and SC Mulholland, editors]. New York: Plenum Press.

Davies J \& Dickerson J (1991) Nutrient Content of Food Portions. Cambridge, UK: Royal Society of Chemistry.

Dobbie JW \& Smith MB (1982) Silicon: its role in medicine and biology. Scott Med J 27, 1-2.

EC Council (1999) Common Organisation of the Market in Wine. Official Journal of the European Communities L179 42, 1-84.

Expert Group on Vitamins and Minerals (2003) Safe Upper Levels for Vitamins and Minerals. London: Food Standards Agency.

Hayward DM \& Parry DW (1980) Scanning electron microscopy of silica deposits in the culms, floral bracts and awns of barley (Hordeum sativum Jess). Ann Bot 46, 541-548.

Hodson MJ \& Sangster AG (1988) Silica deposition in the inflorescence bracts of wheat (Triticum aestivum L.) I. Scanning electron microscopy and light microscopy. Can J Bot 66, 829-838.

Holland B, Welch AA, Unwin ID, Buss DH, Paul AA \& Southgate DAT (1991) McCance and Widdowson's The Composition of Foods, 5th ed. Cambridge, UK: Royal Society of Chemistry.

Hughes C (1987) The Additives Guide. Chichester, UK: John Wiley and Sons.

Iler RK (1979) The Chemistry of Silica. Solubility, Polymerisation, Colloid and Surface Properties, and Biochemistry. New York: John Wiley \& Sons.

Institute of Medicine (2001) Dietary Reference Intakes for Vitamin A, Vitamin K, Arsenic, Boron, Chromium, Copper, Iodine, Iron, Manganese, Molybdenum, Nickel, Silicon, Vanadium and Zinc. Washington, DC: National Academy Press. 
Jugdaohsingh R, Anderson SHC, Tucker KL, Elliott H, Kiel DP, Thompson RPH \& Powell JJ (2002) Dietary silicon intake and absorption. Am J Clin Nutr 75, 887-893.

Jugdaohsingh R, Tucker KL, Qiao N, Cupples LA, Kiel DP \& Powell JJ (2004) Dietary silicon intake is positively associated with bone mineral density in men and premenopausal women of the Framingham Offspring Cohort. J Bone Miner Res 13, 297-307.

Lomer MCE, Hutchinson C, Volkert S, Greenfield SM, Catterall A, Thompson RPH \& Powell JJ (2004) Dietary sources of inorganic microparticles and their intake in healthy subjects and patients with Crohn's disease. Br J Nutr 92, 947-955.

Ministry of Agriculture Fisheries and Foods (1993) Food Portion Sizes, 2nd edition. London: The Stationery Office.

Parry DW, Hodson MJ \& Sangster AG (1984) Some recent advances in studies of silicon in higher plants. Phil Trans $R$ Soc London 304B, 537-549.

Pennington JAT (1991) Silicon in food and diets. Food Addit Contam 8, 97-118.

Powell JJ \& Bolton-Smith C (2003) Interaction between Silicon Intake and Vitamin D and K Status on Markers of Bone Health in Older Women. Final Report to the Food Standards Agency. Project No. 5034. London: Food Standards Agency
Reffitt DM, Jugdaohsingh R, Thompson RPH \& Powell JJ (1999) Silicic acid: its gastrointestinal uptake and urinary excretion in man and effects on aluminium excretion. J Inorg Biochem 76, 141-147.

Saleh MA, Ewane E, Jones J \& Wilson BL (2001) Chemical evaluation of commercial bottled drinking water from Egypt. J Food Comp Anal 14, $127-152$.

Schwarz K (1977) Silicon, fibre and atherosclerosis. Lancet i, 454-457.

Schwarz K \& Milne DB (1972) Growth-promoting effects of silicon in rats. Nature 239, 333-334.

Seaborn CD \& Nielsen FH (1993) Silicon: a nutritional beneficence for bones, brains and blood vessels. Nutr Today 28, 13-18.

Southgate DAT (2000) Vegetables, fruits, funghi and their products. In Human Nutrition and Dietetics, [JS Garrow, WPT James and A Ralph, editors]. London: Churchill-Livingstone.

Sripanyakorn S, Jugdaohsingh R, Elliott H, Walker C, Mehta P, Shouker S, Thompson RPH \& Powell JJ (2004) The silicon content of beer and its bioavailability in healthy volunteers. $\mathrm{Br} J \mathrm{Nutr} \mathbf{9 1}$, 403-409.

van Dyck K, Robberecht H, van Cauwenbergh R, et al. (2000) Spectrometric determination of silicon in food and biological samples: an interlaboratory trial. J Anal At Spectrom 15, 735-741. 